COMMENT. In this study, $27 \%$ of children with complex febrile seizures (CFS) developed epilepsy at follow-up. Of the 3 characteristics that define a CFS (prolonged duration $>15$ minutes, focal partial pattern, or multiple recurrence with a single fever episode), the partial CFS patient carried a higher risk of developing epilepsy (45\%) than patients with multiple seizures (21\%). Only 3 of the 57 patients with CFS had prolonged FS and none developed epilepsy. In some previous studies, the risk of epilepsy following CFS has been lower, reported at 4 15\% (Nelson \& Ellenberg,1976; Verity \& Golding, 1991). Berg and Shinnar (1996) found a strong correlation between prolonged CFS and focal features. In my own prospective studies and reports (Millichap, 1960-1968), prolonged FS, an abnormal $\mathrm{EEG}$, and frequent recurrence of FS were associated with an increased risk of epilepsy. Focal EEG abnormalities were more predictive of epilepsy than focal FS patterns. Spontaneous seizures and epilepsy developed in $29 \%$ of my patients with CFS over a 2 year follow-up, a figure similar to that reported above by Sapir et al, but the prolonged FS duration was more predictive of epilepsy than the focal pattern. For further reference to FS and epilepsy, see Millichap JG, Progress in Pediatric Neurology III, PNB Publ, 1997;pp19-36; and Febrile Convulsions, MacMillan, 1968).

\title{
SEIZURE PRECIPITANTS AND EPILEPSY SYNDROMES
}

The prevalence of various seizure precipitants in relation to different epilepsy syndromes and patient age and gender was determined by a questionnaire survey of 400 patients attending (Dec 1998-May 1999) the Comprehensive Epilepsy Program, University of Virginia, Charlottesville. The mean age of the pediatric and adult patients was $25+\%-16$ years; 200 were male and 200 female. Specific ILAE epilepsy syndromes were defined in 309 (77\%). At least one specific seizure precipitant was identified by $247(62 \%)$ patients, the most frequent being stress, in $30 \%$. Sleep deprivation was invoked by $18 \%$, sleep in $14 \%$, fever or illness (excluding simple febrile convulsions) in 14\%, and fatigue in $13 \%$. Other less frequent precipitants included heat and humidity (9\%), flashing lights (4\%), caffeine (2\%), fasting (2\%), and alcohol in only $0.5 \%$. Rare precipitants were physical discomfort or pain in 3 patients, exertion in 2, awakening (2), light (2), odors (1), laughing (1), noise (1), and dietary triggers (1). No precipitant was recognized by $38 \%$ of patients.

Stress, fatigue, and sleep deprivation were positively correlated, and these precipitants were reported especially in patients with symptomatic localizationrelated epilepsies; $46 \%$ of patients with temporal lobe epilepsy (TLE) identified stress compared to $15 \%$ of those with cryptogenic generalized epilepsy (CGE). Sleep was negatively correlated with other precipitants, and was invoked in $66 \%$ of patients with idiopathic partial, extratemporal epilepsy compared to $4 \%$ of those with TLE. Extralimbic seizures (autosomal dominant frontal lobe epilepsy) are nocturnal and occur especially during sleep whereas limbic seizures (TLE) are diurnal and occur in wakefulness. Menstrual effects were particularly prominent in women with TLE (28\%), more than twice the percentage in women with other epilepsy syndromes. (Frucht MM, Quigg M, Schwaner C, Fountain NB. Distribution of seizure precipitants among epilepsy syndromes. Epilepsia December 2000;41:1534-1539). (Reprints: Dr Mark S Quigg, Department of Neurology, Box 394, Charlottesville, VA 22908).

COMMENT. The recognition and avoidance of specific seizure precipitants is an important aspect of the management of epilepsy. Stress, sleep deprivation, and fatigue are endogenous seizure precipitants that appear to act through common 
mechanisms. Sleep deprivation, usually associated with stress and fatigue, is a frequent trigger in patients with temporal lobe epilepsy whereas sleep predisposes to extratemporal and especially frontal lobe nocturnal epilepsy. Cryptogenic generalized epilepsies are less susceptible to the effects of sleep deprivation.

Numerous reflex epilepsy syndromes are described in children. These include epilepsy induced by "Soroban," a Japanese calculator; reading epilepsy, graphogenic, arithmetic, cards and game playing, piano, Nintendo and Rubik's cube epilepsy. Both mental activity under psychological tension and movements of fingers are involved in the induction of seizures in susceptible patients. (See Progress in Pediatric Neurology II, PNB Publ, 1994;pp60-61).

\section{CHILDHOOD EPILEPSY WITH OCCIPITAL PAROXYSMS:VARIANTS}

The recent ILAE classification of "childhood epilepsy with occipital paroxysms. (CEOP)" into two distinct syndromes was tested at the Schneider Children's Medical Center, Tel Aviv University, and Rappaport Medical School, Haifa, Israel. Of patients with partial-onset seizures and interictal occipital spikes referred to the pediatric seizure unit between Jan 1975 and May 1997, 134 met criteria for CEOP. Three clinical groups were defined: Group 1 (visual) with ictal visual symptoms (24 patients (18\%)); Group 2 (adversive) with tonic eye deviation (72 patients (54\%)); Group 3 (nonvisual, nonadversive) with various seizure patterns (38 patients (28\%). Two syndromes were identified: 1) Gastaut type, late onset (median age 8 years), with brief and frequent diurnal seizures, included the Group 1 visual type; and 2) the Panayiotopoulos type, early onset (median age 5 years), with ictal eye deviation and ictal vomiting, infrequent nocturnal seizures, included the patients in Group 2 adversive type. The patients in Group 3 did not satisfy criteria for either syndrome. (Kivity S, Ephraim T, Weitz R, Tamir A. Childhood epilepsy with occipital paroxysms: clinical variants in 134 patients. Epilepsia Dec 2000;41:1522-1533). (Reprints: Dr S Kivity, Pediatric Epilepsy Unit, Schneider Children's Medical Center of Israel, Petah Tiqva 49202, Israel).

COMMENT. The authors conclude that Panayiotopoulos syndrome is the most common variant of CEOP, and the symptoms are sufficiently delineated to justify classification separate from the Gastaut type CEOP and, also, from BECT, another idiopathic localization-related epilepsy. For all these idiopathic epilepsy syndromes, the prognosis is generally good.

Occipital epileptiform discharges in the EEG can also be associated with symptomatic epilepsies that carry a poor prognosis. Background slowing in the EEG is predictive of persistent seizures and developmental delay (Libenson MH et al, 1999; see Ped Neur Briefs Aug 1999;13:63).

\section{EXCESSIVE BRUISING AND THE KETOGENIC DIET}

Changes in platelet function and excessive bruising were investigated by chart review and prospective screening in 51 patients treated with the ketogenic diet for epilepsy at RUSH-Presbyterian-St Luke's Medical Center, Chicago, IL A significant increase in bruising or other minor bleeding was reported and/or observed in $16(31 \%)$ patients. The complication was more frequent in younger patients but was independent of sex and number of concurrent anticonvulsants (AED). The average age of patients with bleeding was 5 years compared to 8 years for patients without bleeding. The possibility of an interaction with a specific AED was suggested by a $25 \%$ use of lamotrigine in patients with bruises compared to $8 \%$ use in the nonbruising group (not significant $\mathrm{p}=.11$ ). Five of 6 patients tested had prolonged bleeding times and all had diminished response to platelet 